\title{
Inhibitory Effects of Momordicine I on High-Glucose-Induced Cell Proliferation and Collagen Synthesis in Rat Cardiac Fibroblasts
}

\author{
Po-Yuan Chen $\mathbb{D}^{1},{ }^{1}$ Neng-Lang Shih, ${ }^{2}$ Wen-Rui Hao, ${ }^{3}$ Chun-Chao Chen $\mathbb{D}^{3},{ }^{3}$ Ju-Chi Liu $\left(\mathbb{D},{ }^{3,4}\right.$ \\ and Li-Chin Sung $\mathbb{1}^{3,4}$ \\ ${ }^{1}$ Department of Biological Science and Technology, College of Biopharmaceutical and Food Sciences, China Medical University, \\ Taichung 40402, Taiwan \\ ${ }^{2}$ Department of Life Sciences, College of Science, National University of Kaohsiung, Kaohsiung 811, Taiwan \\ ${ }^{3}$ Division of Cardiology, Department of Internal Medicine, Shuang Ho Hospital, Taipei Medical University, \\ New Taipei City 23561, Taiwan \\ ${ }^{4}$ Department of Internal Medicine, School of Medicine, College of Medicine, Taipei Medical University, Taipei 11031, Taiwan
}

Correspondence should be addressed to Li-Chin Sung; 10204@s.tmu.edu.tw

Received 2 July 2018; Accepted 2 September 2018; Published 8 October 2018

Guest Editor: Felipe L. de Oliveira

Copyright ( 2018 Po-Yuan Chen et al. This is an open access article distributed under the Creative Commons Attribution License, which permits unrestricted use, distribution, and reproduction in any medium, provided the original work is properly cited.

\begin{abstract}
Diabetes-associated cardiac fibrosis is a severe cardiovascular complication. Momordicine I, a bioactive triterpenoid isolated from bitter melon, has been demonstrated to have antidiabetic properties. This study investigated the effects of momordicine I on highglucose-induced cardiac fibroblast activation. Rat cardiac fibroblasts were cultured in a high-glucose $(25 \mathrm{mM}) \mathrm{medium}$ in the absence or presence of momordicine I, and the changes in collagen synthesis, transforming growth factor- $\beta 1$ (TGF- $\beta 1$ ) production, and related signaling molecules were assessed. Increased oxidative stress plays a critical role in the development of high-glucose-induced cardiac fibrosis; we further explored momordicine I's antioxidant activity and its effect on fibroblasts. Our data revealed that a high-glucose condition promoted fibroblast proliferation and collagen synthesis and these effects were abolished by momordicine I $(0.3$ and $1 \mu \mathrm{M})$ pretreatment. Furthermore, the inhibitory effect of momordicine I on high-glucoseinduced fibroblast activation may be associated with its activation of nuclear factor erythroid 2-related factor 2 (Nrf2) and the inhibition of reactive oxygen species formation, TGF- $\beta 1$ production, and Smad $2 / 3$ phosphorylation. The addition of brusatol (a selective inhibitor of Nrf2) or Nrf2 siRNA significantly abolished the inhibitory effect of momordicine I on fibroblast activation. Our findings revealed that the antifibrotic effect of momordicine I was mediated, at least partially, by the inhibition of the TGF- $\beta 1 /$ Smad pathway, fibroblast proliferation, and collagen synthesis through Nrf2 activation. Thus, this work provides crucial insights into the molecular pathways for the clinical application of momordicine I for treating diabetes-associated cardiac fibrosis.
\end{abstract}

\section{Introduction}

The prevalence of diabetes mellitus (DM) is rapidly increasing, and cardiovascular complications are the principal causes of morbidity and mortality among patients with DM. The Framingham study reported a 2.4 -fold increase in the incidence of heart failure in diabetic men and a 5-fold increase in diabetic women $[1,2]$. Diabetic cardiomyopathy (DCM), which was first introduced in 1972 by Rubler et al., is one of the most severe irreversible complications of DM [3]. DCM is characterized by myocardial fibrosis, left ventricular hypertrophy, and compromised left ventricular systolic and diastolic functions $[2,4,5]$. Left ventricular diastolic dysfunction is a major characteristic of DCM, and recent studies revealed that over $50 \%$ of asymptomatic diabetic patients have diastolic dysfunction [1, 6]. Cardiac fibroblasts are enmeshed in the myocardium and play a crucial role in maintaining the integrity and homeostasis of the interstitial 
matrix in the adult heart [7-9]. In patients with diabetes, profibrotic myofibroblasts are mainly differentiated from cardiac fibroblasts, resulting in inefficiently organized fibrotic matrices, which can lead to myocardial fibrosis, stiffness, and diastolic dysfunction [4]. However, the mechanisms involved in the pathological changes of DCM are not completely understood. Because fibrosis plays a critical role in the pathology of DCM, we focused on the molecular mechanism involved in cardiac fibrosis in DM $[5,10]$.

Oxidative stress may play a fundamental role in inducing cardiomyopathy in patients with chronic DM [5, 11-14]. Various studies have demonstrated that DM is associated with increased formation of reactive oxygen species (ROS) and a decrease in antioxidant potential [14, 15]. An animal study revealed that ROS could directly induce fibrosis by promoting fibroblast proliferation and collagen synthesis in the setting of DM [15]. The transcription factor nuclear factor erythroid 2-related factor 2 (Nrf2), a basic leucine zipper protein, plays a fundamental role in antioxidant response element- (ARE-) dependent heme oxygenase-1 (HO-1) gene expression. HO-1 is considered a critical endogenous antioxidant and cytoprotective enzyme, which may be upregulated by Nrf2 [16]. The Nrf2/HO-1 signaling pathway has been recognized as the most crucial cellular defense mechanism against oxidative stress [17].

Transforming growth factor- $\beta 1$ (TGF- $\beta 1$ ) is involved in cardiac fibrosis in DCM $[4,10,18,19]$. Small mothers against decapentaplegic (Smad) is an intracellular signal transduction protein of the TGF- $\beta 1$ pathway and is involved in these pathological changes [19]. ROS can induce TGF- $\beta 1$ activation, thus resulting in cardiac fibrosis. Euler demonstrated a reduction in TGF- $\beta 1$ levels in patients treated with $\mathrm{N}$-acetylcysteine (NAC) [20].

Clinical studies have demonstrated that hyperglycemia is a crucial etiologic factor leading to diabetic complications [21]. Diastolic dysfunction is correlated with the degree of hyperglycemia; however, intensive glucose-lowering therapy does not always reduce the risk of DCM $[1,22]$. Oxidative stress may be a target for intervention. Other than DM drugs, Momordica charantia (bitter gourd or bitter melon), a member of the Cucurbitaceae family, has been used to manage hyperglycemia and the early signs of DM since ancient times in some countries [23-26]. Momordicine I (Figure 1(a)), a bioactive saponin and cucurbitane-type triterpenoid, has been isolated from Momordica charantia [27-29]. Momordicine I was reported to be a beneficial natural source of antioxidants [30]. Treatment with momordicine I might have therapeutic potential in improving diabetic myocardial dysfunction. However, little information is available about the effect of momordicine I on the cell proliferation and collagen synthesis of cardiac fibroblasts induced by high glucose.

Thus, we hypothesized that momordicine I is particularly capable of suppressing high-glucose-induced cell proliferation and collagen synthesis in cardiac fibroblasts and therefore decelerates the progression of DCM. The specific aims of this study are to delineate how momordicine I inhibits high-glucose-induced cardiac fibroblast activation through the modulation of ROS, Nrf2/HO- 1 pathway, and TGF- $\beta 1$ / Smad pathway.

\section{Material and Methods}

2.1. Antibodies and Reagents. Dulbecco's modified Eagle's medium (DMEM), fetal calf serum, and tissue culture reagents were obtained from Invitrogen/GIBCO (Grand Island, NY, USA). Momordicine I (>99\% purity, kindly provided by Dr. Shi-Yie Cheng, Department of Life Sciences, College of Science, National University of Kaohsiung, Kaohsiung, Taiwan, ROC) was dissolved in dimethyl sulfoxide (DMSO), and the DMSO content in all groups was 0.1\%. Anti-p-Smad2/3, anti-Smad2/3, anti-GAPDH, and anti-PARP antibodies were purchased from Cell Signaling Technology (Boston, MA, USA). Anti-HO-1 and anti-Nrf2 antibodies were purchased from Santa Cruz Biotechnology (Santa Cruz, CA, USA). Brusatol, the Nrf2-specific inhibitor, and all other reagent-grade chemicals were acquired from Sigma-Aldrich Chemical Co. (St. Louis, MO, USA).

2.2. Culture of Rat Cardiac Fibroblasts. Primary cultures of neonatal rat cardiac fibroblasts were isolated from the hearts of 1-day-old Sprague-Dawley rat pups as previously described [8]. The investigation conformed to the Guide for the Care and Use of Laboratory Animals published by the US National Institutes of Health (NIH publication no. 85-23, revised 1996) and was approved by the Institutional Animal Care and Use Committee of Taipei Medical University. Cardiac fibroblasts grown in culture dishes from the second to fourth passages were used in the experiments and were $>99 \%$ positive for vimentin antibodies (SigmaAldrich). Serum-containing medium from the cultured cells was replaced with serum-free medium, and the cells were subsequently exposed to the treatments as indicated. For fibroblast proliferation assay and TGF- $\beta 1$ secretion assay, following incubation with momordicine I for $12 \mathrm{~h}$, cardiac fibroblasts were then exposed to a serum-free normalglucose medium (5.6 mM glucose) or high-glucose medium (25 mM glucose) for another $24 \mathrm{~h}$ before analyses.

\subsection{3-(4,5-Dimethylthiazol-2-yl)-2,5-diphenyltetrazolium} Bromide (MTT) Assay. MTT assay was used to measure cell viability. After $24 \mathrm{~h}$ incubation with various concentrations of momordicine I as indicated, MTT $(50 \mu \mathrm{L})$ was added and cells were cultured for additional $4 \mathrm{~h}$. Subsequently, cells were lysed using DMSO and the absorbance was measured at $490 \mathrm{~nm}$ by a spectrophotometer.

2.4. Proliferation Assay. Proliferation was assessed through quantifying 5-bromo- 2 '-deoxyuridine (BrdU) incorporation [7]. Cells were removed from the culture dishes by adding trypsin and were subsequently centrifuged. Cell proliferation was assessed through the incorporation of BrdU. BrdU incorporation was determined using a cell proliferation enzyme-linked immunosorbent assay (ELISA) kit (Roche Diagnostics, Mannheim, Germany) in accordance with the manufacturer's instructions.

2.5. ${ }^{3}$ H-Proline Incorporation Assay. Cardiac fibroblasts were incubated using ${ }^{3} \mathrm{H}$-proline $\left(1 \mathrm{mCi} /\right.$ well, $\mathrm{L}$-[2,3,4,5- $\left.{ }^{3} \mathrm{H}\right]$-proline; Amersham Biosciences, Little Chalfont, Buckinghamshire, United Kingdom) for $24 \mathrm{~h}$; subsequently exogenous 


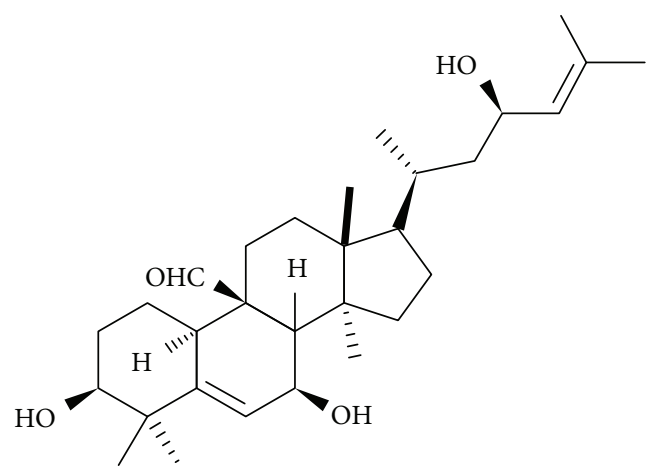

(a)

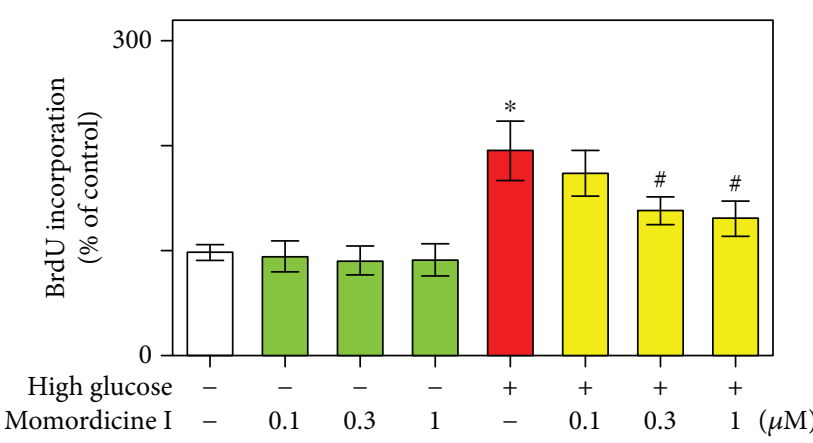

(c)

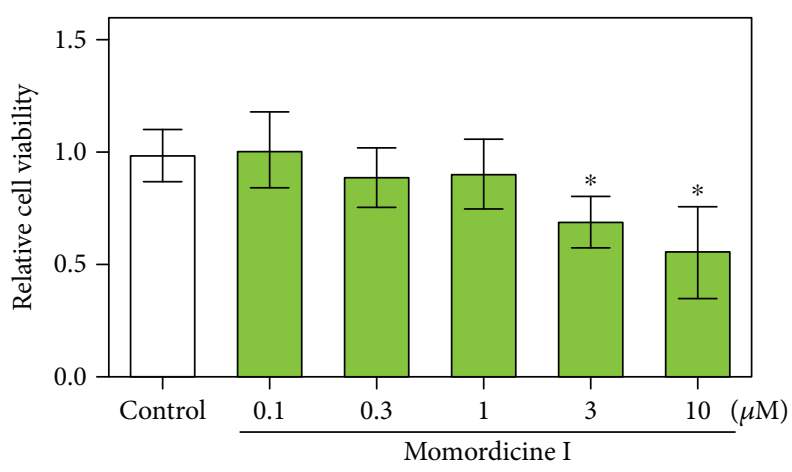

(b)

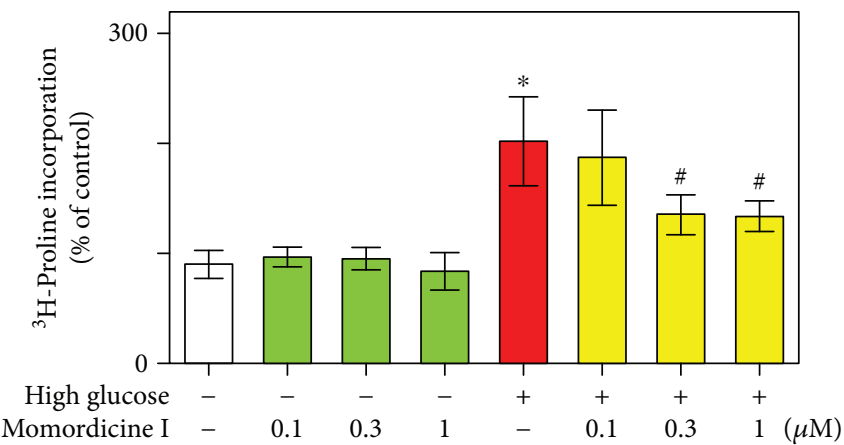

(d)

FIGURE 1: Effects of momordicine I on high-glucose-induced cell proliferation and collagen synthesis in rat cardiac fibroblasts. (a) Chemical structure of momordicine I. (b) Effect of momordicine I $(0.1,0.3,1,3$, and $10 \mu \mathrm{M})$ on cell viability in cardiac fibroblasts. Cell viability was quantified through MTT assay. Effects of momordicine I on high-glucose-stimulated fibroblast proliferation (c) and collagen synthesis (d) were assessed through BrdU and ${ }^{3} \mathrm{H}$-proline incorporation. Rat cardiac fibroblasts were cultured in a serum-free normal-glucose medium (5.6 mM glucose) or high-glucose medium $(25 \mathrm{mM}$ glucose) in the absence or presence of momordicine I $(0.1,0.3$, and $1 \mu \mathrm{M})$ for $24 \mathrm{~h}$. Results were presented as mean \pm SEM $(n=4) .{ }^{*} P<0.05$ versus the control group; ${ }^{\#} P<0.05$ versus the high-glucose group.

${ }^{3} \mathrm{H}$-proline incorporation was determined through scintillation counting.

2.6. Measurement of TGF- $\beta 1$ Concentrations. TGF- $\beta 1$ levels were measured in a culture medium using a commercial ELISA kit (R\&D Systems Inc., Minneapolis, MN, USA) in accordance with the manufacturer's protocols. Results were normalized to cellular protein content in all experiments and expressed as percentages relative to the control group.

2.7. Western Blot Analysis. Nuclear proteins were prepared as previously described [31]. Western blot analysis was performed in accordance with a previously described method [8]. Whole-cell extracts were obtained in a radioimmunoprecipitation assay buffer (Roche Diagnostics GmbH, Germany). Extracts or proteins were separated through sodium dodecyl sulfate polyacrylamide gel electrophoresis followed by electrotransfer to polyvinylidene difluoride membranes and probed with antisera before the introduction of horseradish peroxidase-conjugated secondary antibodies. The proteins were visualized through chemiluminescence in accordance with the manufacturer's instructions (Pierce Biotechnology Inc., Rockford, IL, USA). Images were quantified through densitometry. The densitometry for each band of the phosphorylated form of specific protein (e.g., Smad2/3), the total level of specific protein (e.g., Smad2/3), and loading control (e.g., GAPDH) were measured separately. The specific nonphosphorylated total protein (e.g., Smad2/3) was then normalized against the loading control (e.g., GAPDH) and used the normalized total protein for normalization of the specific phosphoprotein (e.g., Smad2/3). Each control group's value was used to normalize the values of the individual groups obtained in the same experiment. Independent values of control data were normalized on the control mean value, with the control mean value equal to 1 , to generate control values in the bar graph.

2.8. Flow Cytometric Assay of $2^{\prime}, 7^{\prime}$-Dichlorodihydrofluorescein Oxidation. Intracellular ROS production was determined based on the oxidation of $2^{\prime}, 7^{\prime}$-dichlorodihydrofluorescein (DCFH) to a fluorescent $2^{\prime}, 7^{\prime}$-dichlorofluorescein (DCF) as previously described [32]. DCFH was added at a final concentration of $10 \mu \mathrm{M}$ and incubated for $30 \mathrm{~min}$ at $37^{\circ} \mathrm{C}$. Cellular fluorescence was determined through flow cytometry (FACS-SCAN, Becton Dickinson, Franklin Lakes, NJ, USA). Subsequently, the cells were excited using an argon laser operating at $488 \mathrm{nM}$ and measured at $510-540 \mathrm{~nm}$.

2.9. Nrf2 Short Interfering (Si) RNA Transfection. Cardiac fibroblasts were transfected with either Nrf2 siRNA or control siRNA (obtained from Santa Cruz) by using the Lipofectamine reagent as described previously [31]. 
2.10. Statistical Analysis. Results were expressed as mean \pm standard error of the mean (SEM) for at least three experiments unless otherwise stated. Statistical analysis was performed using Student's $t$-test or analysis of variance followed by Tukey's multiple comparisons using GraphPad Prism software (GraphPad Software, San Diego, CA, USA). $P<0.05$ was considered statistically significant.

\section{Results}

3.1. Inhibitory Effect of Momordicine I on High-GlucoseInduced Cell Proliferation and Collagen Synthesis in Rat Cardiac Fibroblasts. Rat cardiac fibroblasts were treated with various concentrations of momordicine I, and cell viability was determined through MTT assay. The effects of momordicine I on cell viability in cultures are presented in Figure 1(b). Momordicine I had no toxic effect on rat cardiac fibroblasts at concentrations of $0.1-1 \mu \mathrm{M}$, so concentrations of $0.1,0.3$, and $1 \mu \mathrm{M}$ momordicine I were used for further analysis. We subsequently tested the effect of momordicine I on cardiac fibroblast proliferation and collagen synthesis. Isolated cardiac fibroblast cells were cultured in normaland high-glucose media. The effects of momordicine I on high-glucose-stimulated cardiac fibroblast proliferation and collagen synthesis were assessed by incorporating BrdU and ${ }^{3} \mathrm{H}$-proline. Compared with culturing in the normal-glucose medium, culturing in the high-glucose $(25 \mathrm{mM})$ medium for $24 \mathrm{~h}$ slightly stimulated fibroblast proliferation and collagen synthesis (Figures 1(c) and 1(d)). Pretreatment of cardiac fibroblasts with momordicine I $(0.3$ and $1 \mu \mathrm{M})$ for $12 \mathrm{~h}$ followed by exposure to a high-glucose medium resulted in a significant decrease in high-glucose-induced cell proliferation and collagen synthesis (Figures 1(c) and 1(d)). These data demonstrate that momordicine I inhibited highglucose-induced cardiac fibroblast activation.

\subsection{Inhibitory Effect of Momordicine I on High-Glucose-} Induced TGF- $\beta 1$ Secretion and Smad2/3 Phosphorylation in Rat Cardiac Fibroblasts. To investigate whether momordicine I affects TGF- $\beta 1$ in cardiac fibroblasts exposed to high-glucose medium, cardiac fibroblasts were treated with momordicine I under high-glucose conditions. TGF- $\beta 1$ secretion was determined through ELISA. As depicted in Figure 2(a), the ELISA results revealed that the cardiac fibroblasts treated with high-glucose medium exhibited increased TGF- $\beta 1$ secretion compared with the cardiac fibroblasts treated with a normal-glucose $(5.6 \mathrm{mM})$ medium. However, high-glucose medium-induced TGF- $\beta 1$ secretion was prevented by treating cardiac fibroblasts with momordicine I $(0.3$ and $1 \mu \mathrm{M})$.

TGF- $\beta$ receptor activation may increase Smad- $2 / 3$ phosphorylation and deploy many of their effects [20]. Therefore, phosphorylated Smad2/3 was also detected. Following incubation with momordicine I $(1 \mu \mathrm{M})$ for $12 \mathrm{~h}$, cells were exposed to a high-glucose medium for $24 \mathrm{~h}$ or humanrecombined TGF- $\beta 1(10 \mathrm{ng} / \mathrm{mL})$ for $2 \mathrm{~h}$. After the aforementioned treatment, total cell proteins were extracted and subjected to Western blotting. As illustrated in Figure 2(b), levels of total Smad2/3 protein in cardiac fibroblasts

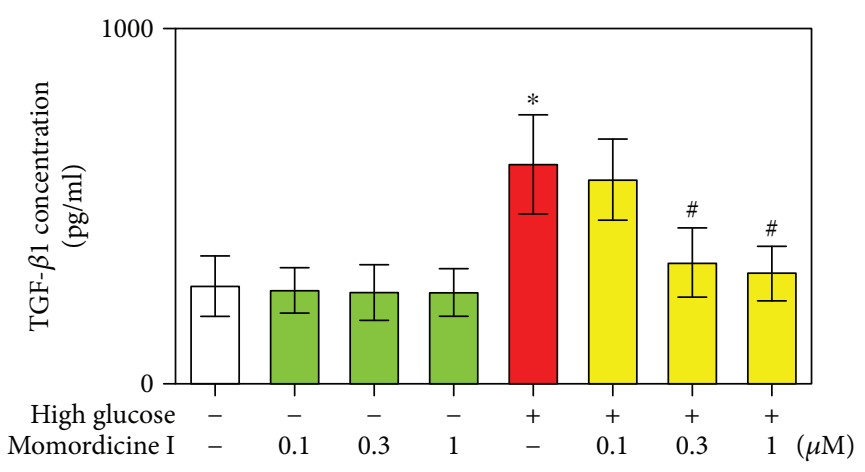

(a)
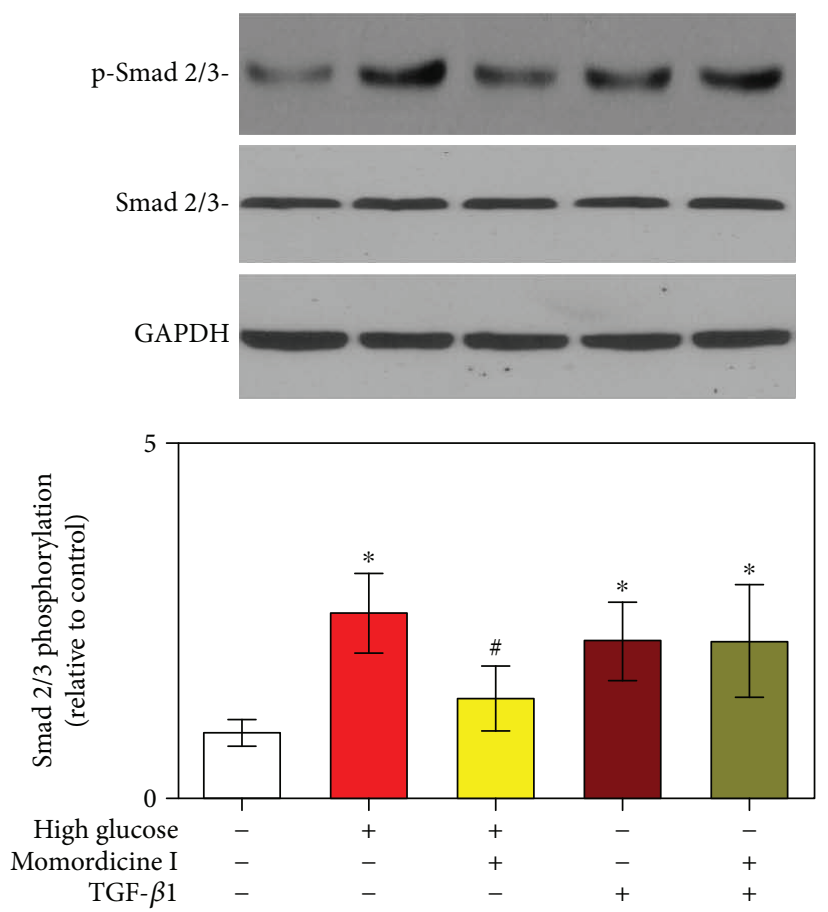

(b)

FIGURE 2: Effects of momordicine I on high-glucose-induced TGF$\beta 1$ secretion and $S m a d 2 / 3$ phosphorylation in rat cardiac fibroblasts. (a) Cardiac fibroblasts treated using a normal-glucose medium or high-glucose medium in the absence or the presence of momordicine I $(0.1,0.3,1 \mu \mathrm{M})$ for $24 \mathrm{~h}$ and the secretion of TGF- $\beta 1$ in cardiac fibroblast supernatants measured through ELISA. Results are presented as mean $\pm \operatorname{SEM}(n=5)$. (b) Effect of momordicine I on high-glucose- or TGF- $\beta 1$-induced Smad2/ 3 phosphorylation. The protein expression levels of Smad2/3 and $\mathrm{p}-\mathrm{Smad} 2 / 3$ were detected using Western blotting following incubation with momordicine I $(1 \mu \mathrm{M})$ for $12 \mathrm{~h}$; cardiac fibroblasts were exposed to high glucose for $24 \mathrm{~h}$ or TGF- $\beta 1$ $(10 \mathrm{ng} / \mathrm{mL})$ for $2 \mathrm{~h}$. Representative micrographs of Smad $2 / 3$ and p-Smad2/3 expression in Western blot analysis (upper) and quantitative results (lower). Results are presented as mean \pm SEM $(n=3) .{ }^{*} P<0.05$ versus the control group; ${ }^{\#} P<0.05$ versus the high-glucose group.

remained unchanged after exposure to a high-glucose medium for $24 \mathrm{~h}$ or TGF- $\beta 1$ for $2 \mathrm{~h}$. However, the level of $\mathrm{p}$-Smad2/3 was notably increased in high-glucose or TGF$\beta 1$-treated cardiac fibroblasts as compared to that in cells 


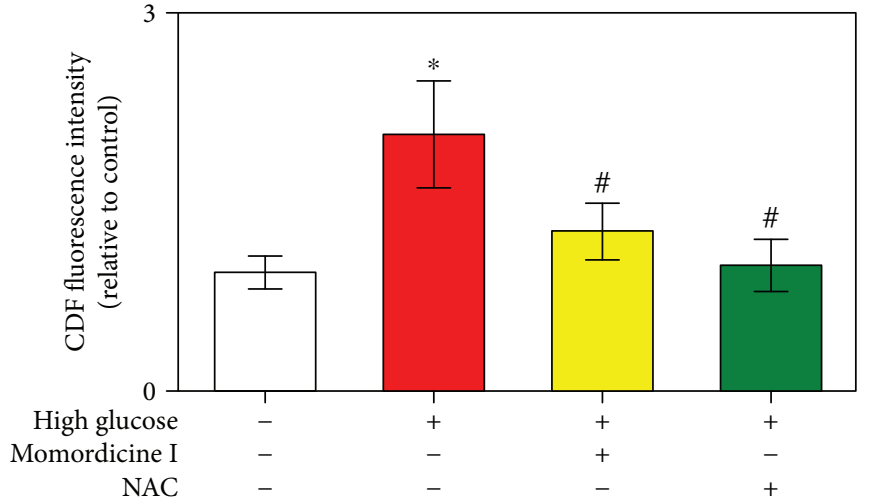

(a)

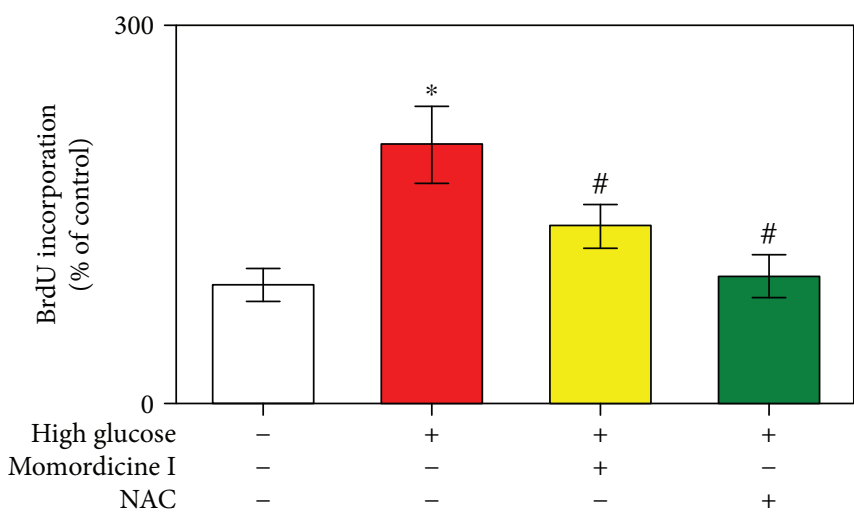

(c)

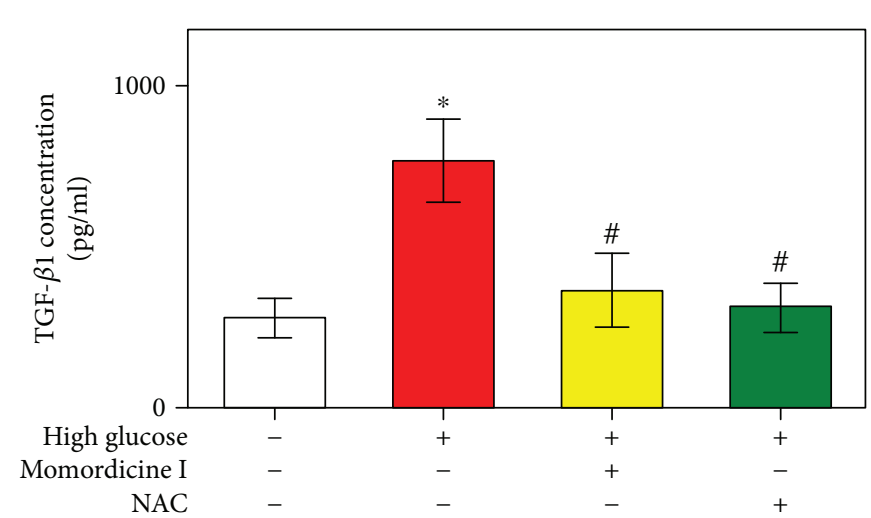

(b)

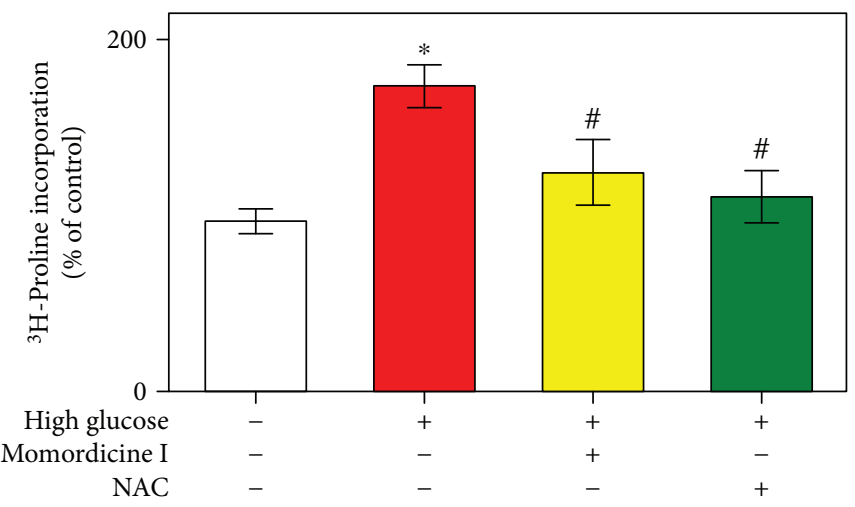

(d)

FIGURE 3: Momordicine I inhibits high-glucose-induced ROS in rat cardiac fibroblasts. (a) Effects of momordicine I on high-glucose-induced ROS generation. Cardiac fibroblasts were cultured in a normal-glucose medium or high-glucose medium for 30 min or preincubated with momordicine I $(1 \mu \mathrm{M}$, for $12 \mathrm{~h})$ or NAC $(5 \mathrm{mM}$, for $30 \mathrm{~min})$ and then stimulated with the high-glucose medium for $30 \mathrm{~min}$. Column bar graph of mean cell fluorescence for DCF. The fluorescence intensities in the control cells are expressed as 100\%. (b) Effects of momordicine I and NAC on the high-glucose-induced secretion of TGF- $\beta 1$ in cardiac fibroblasts. Effects of momordicine I and NAC on high-glucose-induced fibroblast proliferation (c) and collagen synthesis (d). Cardiac fibroblast cells were cultured in the control medium or high-glucose medium in the absence or presence of momordicine I $(1 \mu \mathrm{M})$ or NAC $(5 \mathrm{mM})$ for $24 \mathrm{~h}$. Results are presented as mean \pm SEM $(n=4) .{ }^{*} P<0.05$ versus the control group; ${ }^{\#} P<0.05$ versus the high-glucose group.

treated with a normal-glucose medium. Preincubation with momordicine I $(1 \mu \mathrm{M})$ partially but significantly suppressed high-glucose-induced phosphorylation of Smad2/3 protein. However, momordicine I $(1 \mu \mathrm{M})$ had no significant effect on TGF- $\beta 1$-induced Smad2/3 phosphorylation. These observations indicated that momordicine I may inhibit the high-glucose-mediated fibrotic response by regulating the TGF- $\beta 1 /$ Smad signaling pathway and suggested that momordicine I was able to suppress high-glucose-induced cardiac fibroblast activation through inhibition of TGF- $\beta 1$ secretion, which was independent of its direct effect on the TGF- $\beta 1 /$ Smad signaling pathway.

3.3. Antioxidant Effects of Momordicine I on High-GlucoseInduced ROS Production in Rat Cardiac Fibroblasts. Increased ROS plays a critical role in the development of DM-related cardiac fibrosis $[4,5,11,14,15,19]$. We subsequently explored the role of momordicine I's antioxidant activity in the inhibition of the high-glucose-induced collagen synthesis. The ROS levels were detected from the fluorescence intensity of the ROS, which was analyzed using DCFH-DA assay and a flow cytometer [32]. Our data revealed that the fluorescence intensity enhanced by high glucose was significantly reduced by momordicine I $(1 \mu \mathrm{M})$ (Figure 3(a)). Notably, pretreatment with NAC $(5 \mathrm{mM})$ for $2 \mathrm{~h}$ also substantially alleviated the ROS triggered by the high-glucose medium. As presented in Figure 3(b), cardiac fibroblasts treated with a high-glucose medium also exhibited increased TGF- $\beta 1$ secretion compared with cardiac fibroblasts in the normal-glucose medium. However, highglucose-induced TGF- $\beta 1$ secretion was prevented by preincubation with momordicine I $(1 \mu \mathrm{M})$ or NAC $(5 \mathrm{mM})$. Additionally, we analyzed the effect of momordicine I and NAC on cardiac fibroblast proliferation and collagen synthesis. Compared with the normal-glucose medium, the highglucose medium stimulated fibroblast proliferation, which was significantly blocked by treatment with momordicine I or NAC (Figure 3(c)). In addition to the inhibition of the proliferative effect of the high-glucose medium on cardiac fibroblasts, momordicine I or NAC attenuated the 
collagen synthesis induced by the high-glucose-medium (Figure 3(d)). The effects of momordicine I on the highglucose-induced ROS generation, TGF- $\beta 1$ secretion, and fibroblast activation are similar to the effects of NAC, implicating its possible antioxidant role. These findings suggested that the antioxidant effect of momordicine $\mathrm{I}$ is related to the reduced oxidative stress induced by the high-glucose medium.

3.4. Activation of the Nrf2 Signaling Pathway by Momordicine I in Rat Cardiac Fibroblasts. The Nrf2/HO-1 signaling pathway is also associated with antifibrotic actions $[33,34]$. To more thoroughly understand the possible signaling pathways and mechanisms in the action of momordicine I, we ascertained the expression of Nrf2 and Nrf2 downstream HO-1 in cardiac fibroblasts in high-glucose conditions. Our data revealed that a high-glucose condition slightly elevated the translocation of Nrf2 from the cytoplasm to the nucleus and the enhanced HO-1 expression; however, this modulatory effect was further augmented by momordicine I treatment (Figures 4(a) and 4(b)). These data implicated that momordicine I can activate the Nrf2/HO-1 signaling pathway in cardiac fibroblasts in vitro.

3.5. Nrf2 Inhibitor Brusatol or Nrf2 siRNA Abrogated the Inhibitory Effect of Momordicine I on High-Glucose-Induced TGF- $\beta 1$ Secretion, Cell Proliferation, and Collagen Synthesis in Rat Cardiac Fibroblasts. To further verify the role of Nrf2 in the antifibrotic effect of momordicine I, brusatol, a specific Nrf2 inhibitor [35], was used to determine the role of Nrf2 in the effects of momordicine I on high-glucose medium-induced TGF- $\beta 1$ secretion and cardiac fibroblast activation. Cells were treated with brusatol ( $10 \mathrm{nM}, 30 \mathrm{~min})$, followed by $1 \mu \mathrm{M}$ momordicine I for $12 \mathrm{~h}$, and were subsequently cultured in a high-glucose medium for $24 \mathrm{~h}$. The levels of TGF- $\beta 1$ secretion significantly increased under high-glucose conditions compared with the normal-glucose control group (Figure 5(a)). However, these increases were inhibited by momordicine I. However, brusatol significantly abolished momordicine I's inhibitory effect on high-glucoseinduced TGF- $\beta 1$ secretion. As expected, momordicine I significantly reduced the high-glucose-induced fibroblast proliferation and collagen synthesis, which was also significantly reversed by brusatol (Figures 5(b) and 5(c)). The role of $\mathrm{Nrf2}$ in the inhibition of high-glucose-induced TGF- $\beta 1$ secretion, fibroblast proliferation, and collagen synthesis by momordicine I was also examined by silencing of Nrf2 (Figures 5(d)-5(f)). Cardiac fibroblasts transfected with Nrf2 siRNA $(100 \mathrm{nM})$, followed by treatment with momordicine I $(1 \mu \mathrm{M})$ for $12 \mathrm{~h}$, prevented the inhibitory effect of momordicine I on high-glucose-induced TGF- $\beta 1$ secretion, fibroblast proliferation, and collagen synthesis. In contrast, the control siRNA ( $100 \mathrm{nM})$ failed to block the inhibitory effect of momordicine I. Collectively, these data indicated that momordicine I inhibited TGF- $\beta 1$ pathway activation and proliferation and collagen synthesis of cardiac fibroblasts under a high-glucose condition in an $\mathrm{Nrf}-2$ dependent manner.

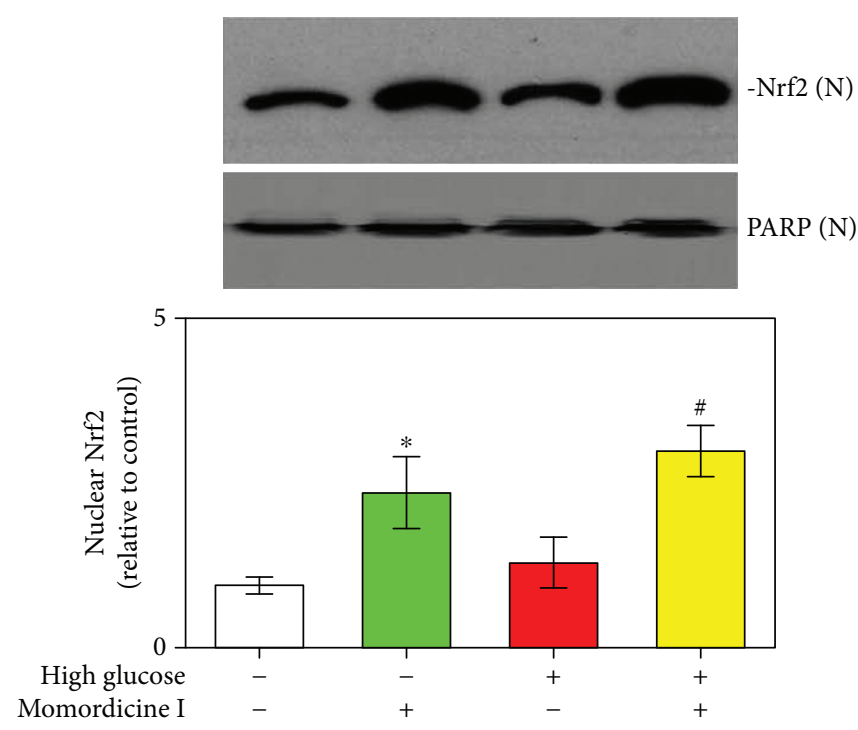

(a)

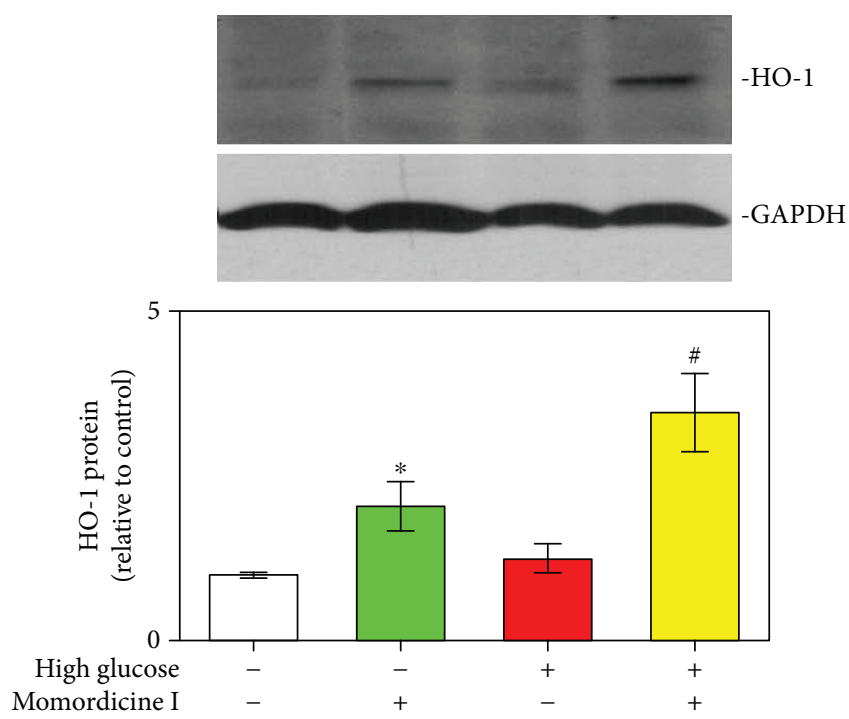

(b)

FIgURE 4: Momordicine I increases Nrf2 translocation and HO-1 protein expression in rat cardiac fibroblasts. (a) Effect of momordicine I on Nrf2 translocation. Cells were treated with or without momordicine I $(1 \mu \mathrm{M})$ for $12 \mathrm{~h}$, followed by a normalglucose medium or high-glucose medium for $12 \mathrm{~h}$. N: nuclear extract. Results are presented as the mean $\pm \operatorname{SEM}(n=4)$. (b) HO-1 expression was determined through Western blotting. Cells were pretreated with momordicine I $(1 \mu \mathrm{M})$ or not for $12 \mathrm{~h}$, followed by the control medium or high-glucose medium for $24 \mathrm{~h}$. Results are presented as the mean \pm SEM $(n=3)$. The relative protein expression of nuclear Nrf2 to PARP and HO-1 to GAPDH are presented in the bar graphs. ${ }^{*} P<0.05$ versus the control group; ${ }^{\#} P<0.05$ versus the high-glucose group.

\section{Discussion}

Through a series of in vitro experiments, we determined that a high-glucose condition led to an increase in cell proliferation, collagen synthesis, and the expression of TGF- $\beta 1$ and Smad2/3 phosphorylation in cardiac fibroblasts. 


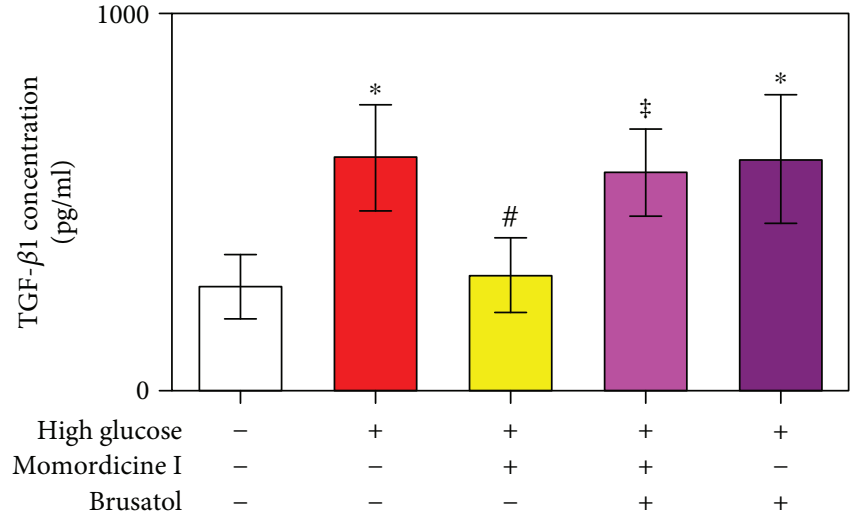

(a)

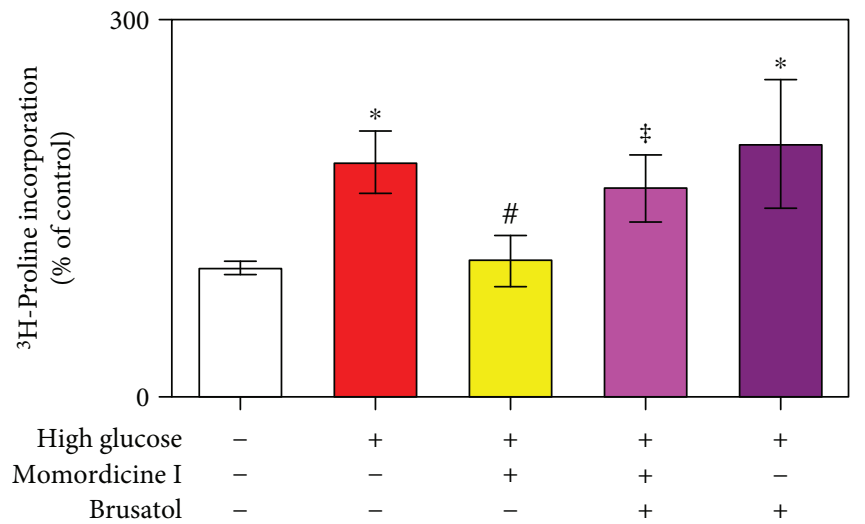

(c)

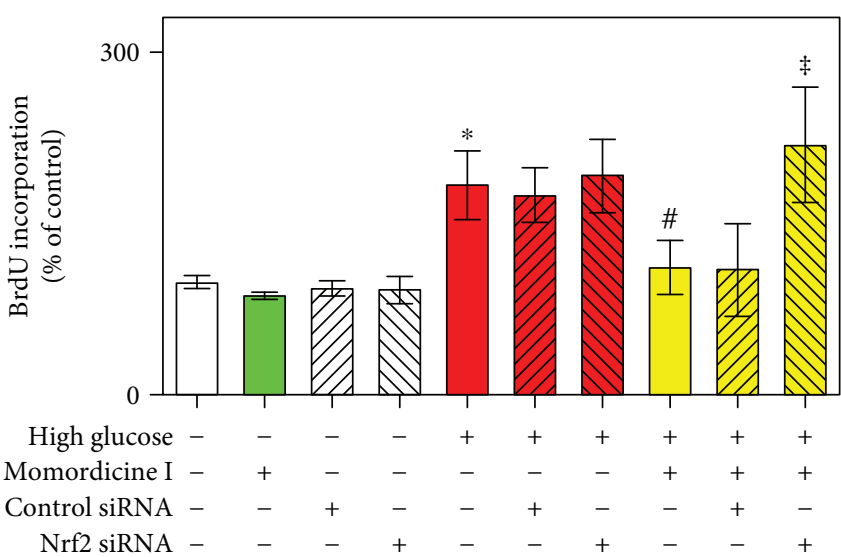

(e)

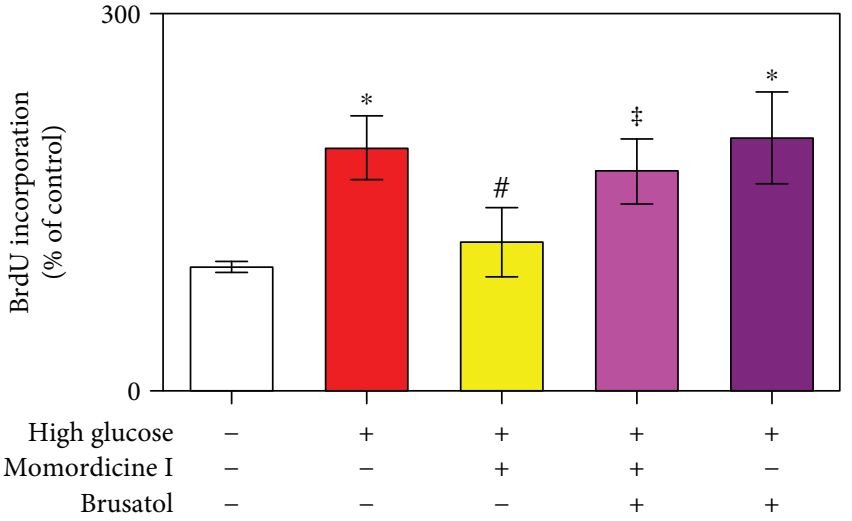

(b)

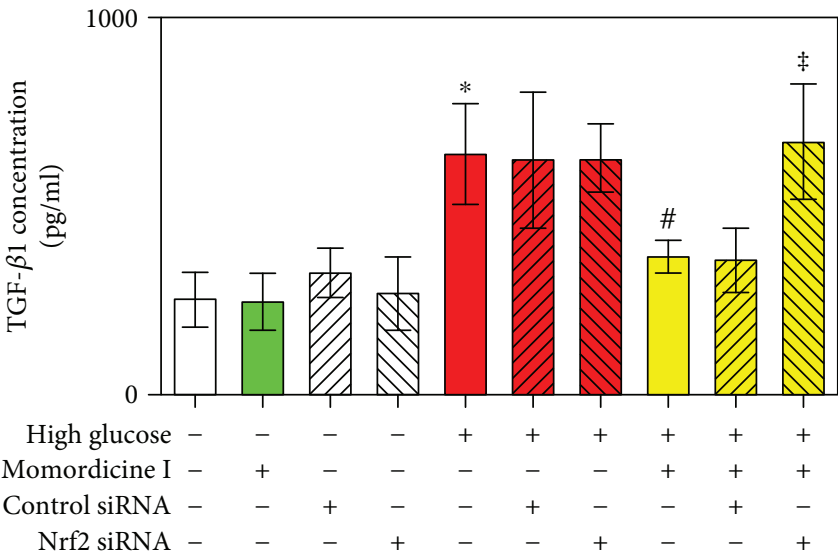

(d)

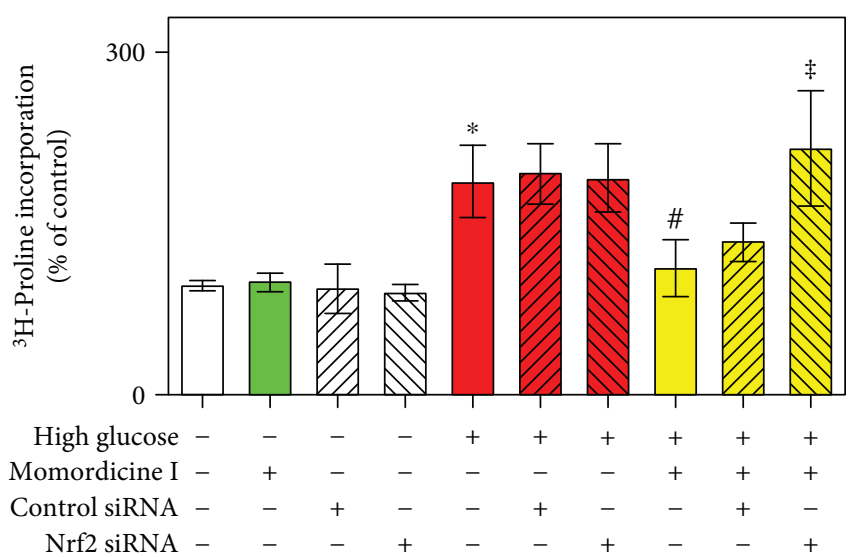

(f)

FIGURE 5: Momordicine I inhibits high-glucose-induced TGF- $\beta 1$ secretion, cell proliferation, and collagen synthesis in rat cardiac fibroblasts in an Nrf2-dependent manner. (a) The Nrf2 inhibitor brusatol prevents the inhibitory effect of momordicine I on high-glucose-induced TGF$\beta 1$ secretion. Results are expressed as means \pm SEM $(n=4)$. (b) Brusatol prevents the inhibitory effect of momordicine I on high-glucoseinduced cell proliferation. Results are expressed as means \pm SEM $(n=4)$. (c) Brusatol prevents the inhibitory effect of momordicine I on high-glucose-induced collagen synthesis. Cells were treated with brusatol $(10 \mathrm{nM})$ for $30 \mathrm{~min}$, followed by $1 \mu \mathrm{M}$ momordicine I for $12 \mathrm{~h}$, and were subsequently cultured in high-glucose medium for $24 \mathrm{~h}$. Results are expressed as means \pm SEM $(n=4)$. (d) Nrf2 siRNA prevents the inhibitory effect of momordicine I on high-glucose-induced TGF- $\beta 1$ secretion. Results are expressed as means \pm SEM ( $n=3$ ). (e) Nrf2 siRNA prevents the inhibitory effect of momordicine I on high-glucose-induced cell proliferation. Results are expressed as means \pm SEM $(n=3)$. (f) Nrf2 siRNA prevents the inhibitory effect of momordicine I on high-glucose-induced collagen synthesis. Results are expressed as means \pm SEM $(n=3)$. ${ }^{*} P<0.05$ compared with the control group; ${ }^{*} P<0.05$ versus the high-glucose group; ${ }^{\ddagger} P<0.05$ versus momordicine I treatment in the high-glucose group. 


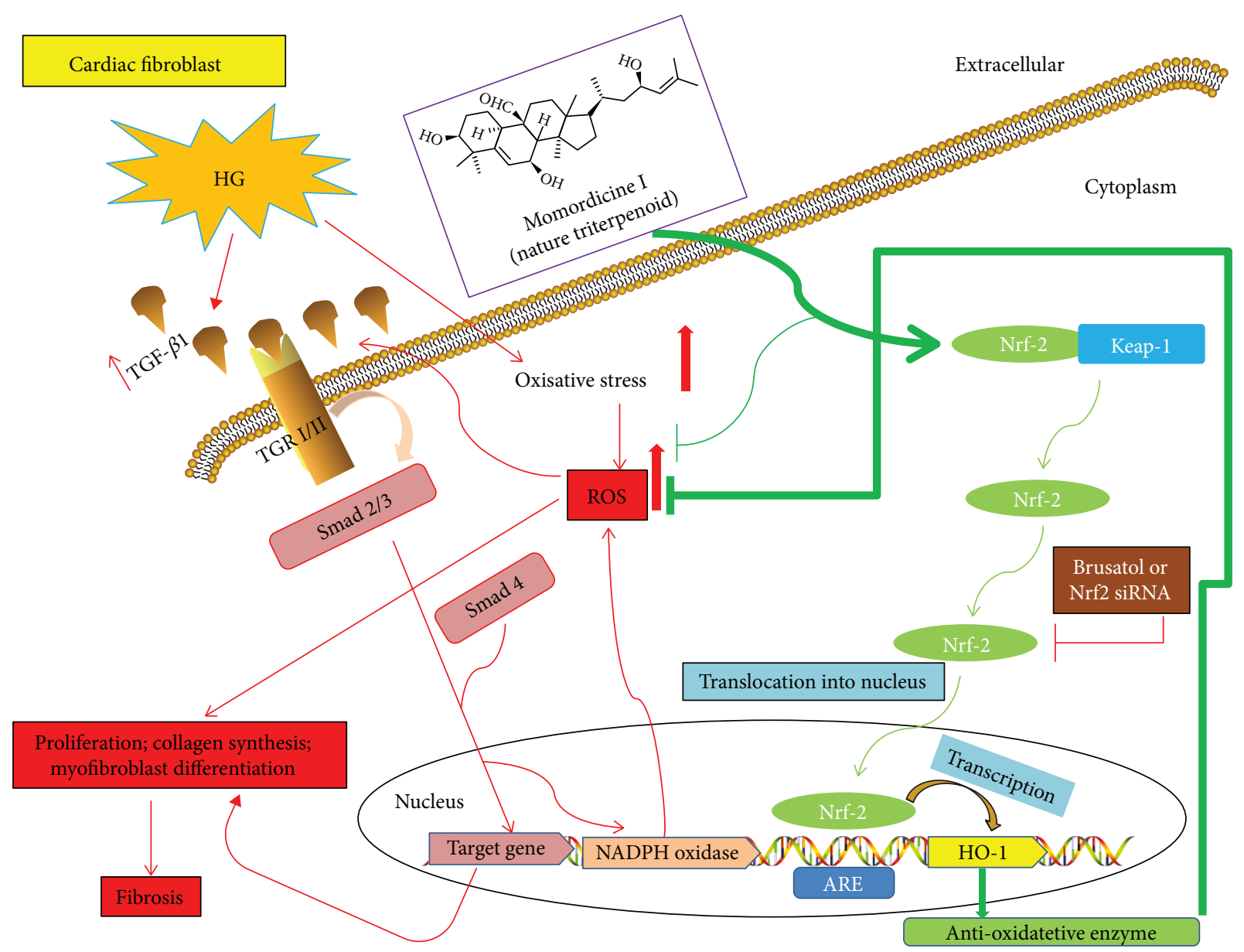

FiguRE 6: Schematic illustration of the proposed mechanism for high-glucose-induced rat cardiac fibroblast activation and momordicine I-inhibited fibrogenesis through the Nrf2-mediated inhibition of oxidative stress and TGF- $\beta 1$ signaling.

Momordicine I pretreatment significantly reduced the high-glucose-induced fibroblast activation by inhibiting the TGF- $\beta 1$-Smad2/3 signaling pathway. We further demonstrated that momordicine I can ameliorate high-glucoseinduced ROS by activating the Nrf2/HO-1 pathway. Furthermore, brusatol (an Nrf2 inhibitor) or Nrf2 siRNA abrogated the inhibitory effect of momordicine I on highglucose-induced TGF- $\beta 1$ secretion. These results suggested that momordicine I inhibits fibrogenesis through the Nrf2mediated modulation of TGF- $\beta 1-\mathrm{Smad} 2 / 3$ signal transduction (Figures 6).

Consumption of food containing antioxidants has been revealed to protect against diseases such as cancer, cardiovascular diseases, and DM [25, 36]. Momordica charantia, commonly known as bitter melon, is a climbing perennial characterized by an elongated, warty fruit-like gourd and has been reported to have medicinal qualities in treating DM [25, 30, 37-39]. A principal therapeutic constituent of bitter melon known as momordicine has long been used in traditional Asian medicine [30]. Momordicine I belongs to cucurbitane-type triterpenoids, the major bioactive components of bitter melon. These have anticancer, antioxidant, antidiabetic, hypoglycemic, and anti-inflammatory properties [27, 30, 39-41]. With literature review and our study, we suppose that momordicine I may be used as complementary treatment targeting cardiac fibrosis in patients with DM.

The pathophysiological mechanisms of DCM are multifactorial; substantial evidence from both clinical data and animal models has indicated that increased cardiac inflammation, oxidative stress, and enhanced cardiac fibrosis contribute to the development of DCM [42]. Therefore, anti-inflammatory, antioxidant, and antifibrotic therapeutic approaches may be beneficial for treating DCM. Fibrogenesis is the most critical factor in the progression of DCM. However, specific pharmaceuticals directly targeting fibrosis are still lacking. In this study, despite clearly acting as an antifibrotic result, momordicine I has been shown to demonstrate antioxidant effects. Furthermore, extensive evidence has indicated that oxidative stress mediates the initiation and development of fibrosis in relation to chronic inflammation [18]. Although our main focus was the modification of ROS levels as a mediator of downstream high-glucoseinduced TGF- $\beta 1$ signaling [20], we discovered that momordicine I alleviated the production of hyperglycemia-induced ROS and might block the activation of high-glucoseinduced cardiac fibroblast by inhibiting ROS and its downstream TGF- $\beta 1$ signal. Additionally, studies have indicated that TGF- $\beta 1$ increases ROS generation by inducing NADPH oxidase and suppressing antioxidant enzymes; in turn, ROS 
activates TGF- $\beta 1$ signaling and mediates several of its fibrogenic effects, in a vicious cycle $[2,15,18,20]$. These results further strengthen the evidence for the antioxidant properties of momordicine I through its inhibition of ROS. To confirm the role of oxidative stress in high-glucoseinduced cardiac fibroblast activation, we treated cardiac fibroblasts with the antioxidant NAC. Similarly, the ROS scavenger NAC also decreased high-glucose-induced cardiac fibroblast activation. However, whether any antioxidant could have the beneficial effects of momordicine I in decreasing high-glucose-induced cardiac fibroblast activation remains to be examined in future studies.

The TGF- $\beta$ family of growth factors is the most extensively studied mediator of fibroblast activation, of which TGF- $\beta 1$ likely plays a crucial role in pathological fibrosis [9]. The profibrotic actions of TGF- $\beta 1$ on cardiac fibroblasts are mediated, at least partially, by Smad3 [20, 43, 44]. Bujak et al. demonstrated that the loss of Smad3 prevents interstitial fibrosis in the noninfarcted remodeling of the myocardium [44]. TGF- $\beta 1 /$ Smad signaling appears to be responsible for cardiac fibrosis [20]. The canonical pathway of TGF- $\beta 1$ signaling involves the phosphorylation of Smad2/3, which subsequently binds to Smad4 and translocates to the nucleus. Here, transcriptional reprogramming is conducted to promote myofibroblast formation and extracellular matrix production, eventually leading to cardiac fibrosis $[9,20,45]$. Our paper suggests that momordicine I at nontoxic concentrations $(0.1-1 \mu \mathrm{M})$ effectively attenuates the profibrogenic TGF- $\beta 1-S m a d 2 / 3$ signaling pathway and is thus an effective therapy for diabetes-associated cardiac fibrosis. Several natural compounds, including matrine, resveratrol, and tanshinone, also suppressed cardiac fibrosis by inhibiting the TGF- $\beta /$ Smad pathway [46-48].

Under normal conditions, Nrf2 is retained within the cytoplasm, forming a complex with its protein inhibitor Keap1 [36]. On activation, Keap1 cysteine residues undergo oxidoreduction resulting in the release of Nrf2, which subsequently translocates into the nucleus where it binds to ARE and controls the transcription of genes encoding antioxidant enzymes [49]. Nrf2 governs innate immune, antioxidant, and cytoprotective responses, and its deregulation is pivotal in the chronic inflammatory status [33]. Nrf2 has long been recognized to resist oxygen-free radicals and reduce ROS in the alleviation of cardiac fibrosis [34]. A study reported that the forced expression of Nrf2 in normal fibroblasts resulted in the abrogated stimulation of collagen synthesis, myofibroblast differentiation, and ROS generation through the disruption of canonical TGF- $\beta 1$ signaling [33]. Our study revealed that momordicine I not only ameliorates high-glucoseinduced ROS but also effectively promotes Nrf2 translocation to the nucleus, which in turn upregulates $\mathrm{HO}-1$. In this study, by using a selective Nrf2 inhibitor or Nrf2 knockdown, we confirmed that the antifibrotic effects of momordicine I on cardiac fibroblasts under high-glucose conditions were Nrf2 dependent. He et al. investigated the role of Nrf2 in the development of DCM using Nrf2-knockout mice. There was an increased level of ROS in the cardiomyocytes of Nrf2-knockout mice [50]. Notably, Nrf2 attenuated dystrophic muscle fibrosis by inhibiting the TGF- $\beta 1 / \mathrm{Smad}$ pathway [51]. Our study demonstrated that brusatol or Nrf2 siRNA significantly reversed the inhibitory effect of momordicine I on high-glucose-induced fibroblast activation and TGF- $\beta 1$ expression. Thus, momordicine I might reduce high-glucose-induced TGF- $\beta 1$ expression, cell proliferation, and collagen synthesis through the direct antifibrotic effects of Nrf2 and indirect downregulation of ROS levels. However, the exact molecular mechanism of Nrf2-mediated gene regulation in cardiac fibroblasts under high-glucose conditions by momordicine I remains unknown and warrants further investigation. Moreover, the induction of $\mathrm{HO}-1$ is widely recognized as an effective cellular strategy to counteract a variety of cellular damage and inflammation. These effects may be mediated by multiple functions of HO-1. Although the exact mechanisms involved in the antifibrotic effects of HO-1 have not been fully elucidated, momordicine I, as confirmed in this study, can induce the expression of HO- 1 in cardiac fibroblasts; the observed antifibrotic effects of momordicine I might be mediated, at least in part, by one or more of HO-1 by-products. However, whether inhibition of HO-1 activity could block momordicine I-induced antifibrotic action to suggest the role of HO-1 on the antifibrotic effect of momordicine I in cardiac fibroblasts remains to be elucidated.

A limitation of our study is that the antifibrotic efficacy of momordicine I was determined using a single TGF- $\beta 1$ dependent in vitro fibrosis model. Furthermore, the momordicine I-reduced high-glucose-induced collagen production from its inhibitory effect on proliferation cannot be excluded. The exact mechanism and potential role of momordicine I in fibroblast collagen synthesis remain to be investigated. The present in vitro model may not be directly translatable to clinical investigations of DCM. However, our preliminary results may encourage further research on determining the molecular mechanisms underlying high-glucose-induced fibroblast activation and cardiac fibrosis. While these results are promising, a lot of work needs to be done in elucidating the signal pathways involved in the action of momordicine I on these cells. Moreover, in vivo experiments in animal models are essential to establish the validity of these in vitro results in the future.

\section{Conclusions}

On the basis of this study, we propose that through Nrf2 activation, momordicine I can partially block intracellular TGF- $\beta 1$ signal transduction and inhibit the proliferation and collagen synthesis of cardiac fibroblasts. Through this activity, momordicine I reduces collagen synthesis and TGF- $\beta 1$ expression induced by high-glucose treatment, suggesting its potential for clinical application in preventing and treating diabetic myocardial fibrosis. Our study demonstrated that momordicine I attenuates high-glucose-induced fibroblast activation and its antifibrotic effects, at least partially, appear to reduce the expression of the profibrogenic cytokine TGF- $\beta 1$ and inhibit TGF- $\beta 1-$ Smad $2 / 3$ signaling. This suggests that momordicine I has therapeutic potential in treating diabetes-induced cardiac fibrosis. 


\section{Data Availability}

The data of materials and methods and conclusions to support the findings of this study are included within the article. If any other data may be needed, please contact the corresponding author upon request.

\section{Conflicts of Interest}

The authors declare that there is no conflict of interests.

\section{Authors' Contributions}

Po-Yuan Chen and Li-Chin Sung conceived and designed the experiments, Wen-Rui Hao and Po-Yuan Chen performed the experiments, Wen-Rui Hao, Chun-Chao Chen, Ju-Chi Liu, and Li-Chin Sung analyzed the data, Neng-Lang Shih and Li-Chin Sung contributed the reagents and materials, and Po-Yuan Chen and Li-Chin Sung wrote the paper. All authors read and approved the final manuscript.

\section{Acknowledgments}

This study was supported in part by research grants from Taipei Medical University (TMU105-AE1-B21), Taipei, Taiwan.

\section{References}

[1] T. H. Marwick, R. Ritchie, J. E. Shaw, and D. Kaye, "Implications of underlying mechanisms for the recognition and management of diabetic cardiomyopathy," Journal of the American College of Cardiology, vol. 71, no. 3, pp. 339-351, 2018.

[2] J. Tian, Y. Zhao, Y. Liu, Y. Liu, K. Chen, and S. Lyu, "Roles and mechanisms of herbal medicine for diabetic cardiomyopathy: current status and perspective," Oxidative Medicine and Cellular Longevity, vol. 2017, Article ID 8214541, 15 pages, 2017.

[3] T. Miki, S. Yuda, H. Kouzu, and T. Miura, "Diabetic cardiomyopathy: pathophysiology and clinical features," Heart Failure Reviews, vol. 18, no. 2, pp. 149-166, 2013.

[4] N. Shen, X. Li, T. Zhou et al., "Shensong Yangxin capsule prevents diabetic myocardial fibrosis by inhibiting TGF- $\beta 1 /$ Smad signaling," Journal of Ethnopharmacology, vol. 157, pp. 161-170, 2014.

[5] X. T. Wang, Y. Gong, B. Zhou et al., "Ursolic acid ameliorates oxidative stress, inflammation and fibrosis in diabetic cardiomyopathy rats," Biomedicine \& Pharmacotherapy, vol. 97, pp. 1461-1467, 2018.

[6] J. S. Felício, C. C. Koury, C. T. Carvalho et al., "Present insights on cardiomyopathy in diabetic patients," Current Diabetes Reviews, vol. 12, no. 4, pp. 384-395, 2016.

[7] J. Y. Liou, Y. L. Chen, S. H. Loh et al., "Magnolol depresses urotensin-II-induced cell proliferation in rat cardiac fibroblasts," Clinical and Experimental Pharmacology \& Physiology, vol. 36, no. 7, pp. 711-716, 2009.

[8] T. H. Cheng, P. Y. Cheng, N. L. Shih, I. B. Chen, D. L. Wang, and J. J. Chen, "Involvement of reactive oxygen species in angiotensin II-induced endothelin-1 gene expression in rat cardiac fibroblasts," Journal of the American College of Cardiology, vol. 42, no. 10, pp. 1845-1854, 2003.
[9] J. G. Travers, F. A. Kamal, J. Robbins, K. E. Yutzey, and B. C. Blaxall, "Cardiac fibrosis: the fibroblast awakens," Circulation Research, vol. 118, no. 6, pp. 1021-1040, 2016.

[10] Y. Yue, K. Meng, Y. Pu, and X. Zhang, “Transforming growth factor beta (TGF- $\beta$ ) mediates cardiac fibrosis and induces diabetic cardiomyopathy," Diabetes Research and Clinical Practice, vol. 133, pp. 124-130, 2017.

[11] H. Wu, G. N. Li, J. Xie et al., "Resveratrol ameliorates myocardial fibrosis by inhibiting ROS/ERK/TGF- $\beta$ /periostin pathway in STZ-induced diabetic mice," BMC Cardiovascular Disorders, vol. 16, no. 1, p. 5, 2016.

[12] Y. J. Xu, P. S. Tappia, N. S. Neki, and N. S. Dhalla, "Prevention of diabetes-induced cardiovascular complications upon treatment with antioxidants," Heart Failure Reviews, vol. 19, no. 1, pp. 113-121, 2014.

[13] A. Ceriello and E. Motz, "Is oxidative stress the pathogenic mechanism underlying insulin resistance, diabetes, and cardiovascular disease? The common soil hypothesis revisited," Arteriosclerosis, Thrombosis, and Vascular Biology, vol. 24, no. 5, pp. 816-823, 2004.

[14] D. Zheng, S. Dong, T. Li et al., "Exogenous hydrogen sulfide attenuates cardiac fibrosis through reactive oxygen species signal pathways in experimental diabetes mellitus models," Cellular Physiology and Biochemistry, vol. 36, no. 3, pp. 917929, 2015.

[15] C. Liu, X. Z. Lu, M. Z. Shen et al., "N-acetyl cysteine improves the diabetic cardiac function: possible role of fibrosis inhibition," BMC Cardiovascular Disorders, vol. 15, no. 1, p. 84, 2015.

[16] Z. Wang, H. Zhang, X. Sun, and L. Ren, "The protective role of vitamin D3 in a murine model of asthma via the suppression of TGF- $\beta /$ Smad signaling and activation of the Nrf $2 / \mathrm{HO}-1$ pathway," Molecular Medicine Reports, vol. 14, no. 3, pp. 23892396, 2016.

[17] W. J. Bae, G. Q. Zhu, S. W. Choi et al., "Antioxidant and antifibrotic effect of a herbal formulation in vitro and in the experimental andropause via Nrf 2/HO-1 signaling pathway," Oxidative Medicine and Cellular Longevity, vol. 2017, Article ID 6024839, 10 pages, 2017.

[18] G. Latella, "Redox imbalance in intestinal fibrosis: beware of the TGF $\beta-1$, ROS, and Nrf 2 connection," Digestive Diseases and Sciences, vol. 63, no. 2, pp. 312-320, 2018.

[19] X. Liu, X. Song, J. Lu et al., "Neferine inhibits proliferation and collagen synthesis induced by high glucose in cardiac fibroblasts and reduces cardiac fibrosis in diabetic mice," Oncotarget, vol. 7, no. 38, pp. 61703-61715, 2016.

[20] G. Euler, "Good and bad sides of TGF $\beta$-signaling in myocardial infarction," Frontiers in Physiology, vol. 6, p. 66, 2015.

[21] H. Y. Lo, T. Y. Ho, C. C. Li, J. C. Chen, J. J. Liu, and C. Y. Hsiang, "A novel insulin receptor-binding protein from Momordica charantia enhances glucose uptake and glucose clearance in vitro and in vivo through triggering insulin receptor signaling pathway," Journal of Agricultural and Food Chemistry, vol. 62, no. 36, pp. 8952-8961, 2014.

[22] H. Yamada, A. Tanaka, K. Kusunose et al., "Effect of sitagliptin on the echocardiographic parameters of left ventricular diastolic function in patients with type 2 diabetes: a subgroup analysis of the PROLOGUE study," Cardiovascular Diabetology, vol. 16, no. 1, p. 63, 2017.

[23] C. Ma, H. Yu, Y. Xiao, and H. Wang, "Momordica charantia extracts ameliorate insulin resistance by regulating the 
expression of SOCS-3 and JNK in type 2 diabetes mellitus rats," Pharmaceutical Biology, vol. 55, no. 1, pp. 2170-2177, 2017.

[24] A. Raman and C. Lau, "Anti-diabetic properties and phytochemistry of Momordica charantia L. (Cucurbitaceae)," Phytomedicine, vol. 2, no. 4, pp. 349-362, 1996.

[25] B. C. Panda, S. Mondal, K. S. P. Devi et al., "Pectic polysaccharide from the green fruits of Momordica charantia (Karela): structural characterization and study of immunoenhancing and antioxidant properties," Carbohydrate Research, vol. 401, pp. 24-31, 2015.

[26] H. Z. Gu, R. R. Lin, H. C. Wang, X. J. Zhu, Y. Hu, and F. Y. Zheng, "Effect of Momordica charantia protein on proliferation, apoptosis and the AKT signal transduction pathway in the human endometrial carcinoma Ishikawa $\mathrm{H}$ cell line in vitro," Oncology Letters, vol. 13, no. 5, pp. 3032-3038, 2017.

[27] S.-B. Wu, G. G. L. Yue, M.-H. To, A. C. Keller, C. B. S. Lau, and E. J. Kennelly, "Transport in Caco-2 cell monolayers of antidiabetic cucurbitane triterpenoids from Momordica charantia fruits," Planta Medica, vol. 80, no. 11, pp. 907-911, 2014.

[28] M. J. Tan, J. M. Ye, N. Turner et al., "Antidiabetic activities of triterpenoids isolated from bitter melon associated with activation of the AMPK pathway," Chemistry \& Biology, vol. 15, no. 3, pp. 263-273, 2008.

[29] A. C. Keller, J. Ma, A. Kavalier, K. He, A. M. B. Brillantes, and E. J. Kennelly, "Saponins from the traditional medicinal plant Momordica charantia stimulate insulin secretion in vitro," Phytomedicine, vol. 19, no. 1, pp. 32-37, 2011.

[30] S. Jia, M. Shen, F. Zhang, and J. Xie, "Recent advances in Momordica charantia: functional components and biological activities," International Journal of Molecular Sciences, vol. 18 , no. $12,2017$.

[31] S. Zhuang, T. H. Cheng, N. L. Shih et al., "Tanshinone IIA induces heme oxygenase 1 expression and inhibits cyclic strain-induced interleukin 8 expression in vascular endothelial cells," The American Journal of Chinese Medicine, vol. 44, no. 2, pp. 377-388, 2016.

[32] J. C. Liu, C. H. Chen, J. J. Chen, and T. H. Cheng, "Urotensin II induces rat cardiomyocyte hypertrophy via the transient oxidization of Src homology 2-containing tyrosine phosphatase and transactivation of epidermal growth factor receptor," Molecular Pharmacology, vol. 76, no. 6, pp. 1186-1195, 2009.

[33] J. Wei, H. Zhu, G. Lord et al., "Nrf 2 exerts cell-autonomous antifibrotic effects: compromised function in systemic sclerosis and therapeutic rescue with a novel heterocyclic chalcone derivative," Translational Research, vol. 183, pp. 71-86.e1, 2017.

[34] S. M. Swamy, N. S. Rajasekaran, and V. J. Thannickal, "Nuclear factor-erythroid-2-related factor 2 in aging and lung fibrosis," The American Journal of Pathology, vol. 186, no. 7, pp. 1712-1723, 2016.

[35] D. Ren, N. F. Villeneuve, T. Jiang et al., "Brusatol enhances the efficacy of chemotherapy by inhibiting the Nrf2-mediated defense mechanism," Proceedings of the National Academy of Sciences of the United States of America, vol. 108, no. 4, pp. 1433-1438, 2011.

[36] L. C. Sung, H. H. Chao, C. H. Chen et al., "Lycopene inhibits cyclic strain-induced endothelin-1 expression through the suppression of reactive oxygen species generation and induction of heme oxygenase-1 in human umbilical vein endothelial cells," Clinical and Experimental Pharmacology \& Physiology, vol. 42, no. 6, pp. 632-639, 2015.
[37] M. F. Mahmoud, F. E. El Ashry, N. N. El Maraghy, and A. Fahmy, "Studies on the antidiabetic activities of Momordica charantia fruit juice in streptozotocin-induced diabetic rats," Pharmaceutical Biology, vol. 55, no. 1, pp. 758-765, 2017.

[38] S. J. Yang, J. M. Choi, S. E. Park et al., "Preventive effects of bitter melon (Momordica charantia) against insulin resistance and diabetes are associated with the inhibition of NF- $\kappa \mathrm{B}$ and JNK pathways in high-fat-fed OLETF rats," The Journal of Nutritional Biochemistry, vol. 26, no. 3, pp. 234-240, 2015.

[39] A. Ota and N. P. Ulrih, "An overview of herbal products and secondary metabolites used for management of type two diabetes," Frontiers in Pharmacology, vol. 8, p. 436, 2017.

[40] J. Ma, P. Whittaker, A. Keller et al., "Cucurbitane-type triterpenoids from Momordica charantia," Planta Medica, vol. 76, no. 15, pp. 1758-1761, 2010.

[41] H. Liu, G. C. Wang, M. X. Zhang, and B. Ling, "The cytotoxicology of momordicins I and II on Spodoptera litura cultured cell line SL-1," Pesticide Biochemistry and Physiology, vol. 122, pp. 110-118, 2015.

[42] L. M. D. Delbridge, V. L. Benson, R. H. Ritchie, and K. M. Mellor, "Diabetic cardiomyopathy: the case for a role of fructose in disease etiology," Diabetes, vol. 65, no. 12, pp. 3521-3528, 2016.

[43] M. Bujak and N. G. Frangogiannis, "The role of TGF- $\beta$ signaling in myocardial infarction and cardiac remodeling," Cardiovascular Research, vol. 74, no. 2, pp. 184-195, 2007.

[44] M. Bujak, G. Ren, H. J. Kweon et al., "Essential role of Smad 3 in infarct healing and in the pathogenesis of cardiac remodeling," Circulation, vol. 116, no. 19, pp. 2127-2138, 2007.

[45] G. Liu, C. Ma, H. Yang, and P. Y. Zhang, "Transforming growth factor $\beta$ and its role in heart disease," Experimental and Therapeutic Medicine, vol. 13, no. 5, pp. 2123-2128, 2017.

[46] C.-Y. Zhan, J.-H. Tang, D.-X. Zhou, and Z.-H. Li, "Effects of tanshinone IIA on the transforming growth factor $\beta 1 / \mathrm{Smad}$ signaling pathway in rat cardiac fibroblasts," Indian Journal of Pharmacology, vol. 46, no. 6, pp. 633-638, 2014.

[47] T. Chen, J. Li, J. Liu et al., "Activation of SIRT3 by resveratrol ameliorates cardiac fibrosis and improves cardiac function via the TGF- $\beta$ /Smad 3 pathway," American Journal of Physiology. Heart and Circulatory Physiology, vol. 308, no. 5, pp. H424H434, 2015.

[48] Y. Zhang, L. Cui, G. Guan et al., "Matrine suppresses cardiac fibrosis by inhibiting the TGF- $\beta /$ Smad pathway in experimental diabetic cardiomyopathy," Molecular Medicine Reports, vol. 17, no. 1, pp. 1775-1781, 2018.

[49] Q. Ma, "Role of Nrf2 in oxidative stress and toxicity," Annual Review of Pharmacology and Toxicology, vol. 53, no. 1, pp. 401-426, 2013.

[50] X. He, H. Kan, L. Cai, and Q. Ma, "Nrf2 is critical in defense against high glucose-induced oxidative damage in cardiomyocytes," Journal of Molecular and Cellular Cardiology, vol. 46, no. 1, pp. 47-58, 2009.

[51] C. Sun, S. Li, and D. Li, "Sulforaphane mitigates muscle fibrosis in $m d x$ mice via Nrf2-mediated inhibition of TGF- $\beta / \mathrm{Smad}$ signaling," Journal of Applied Physiology, vol. 120, no. 4, pp. 377-390, 2016. 


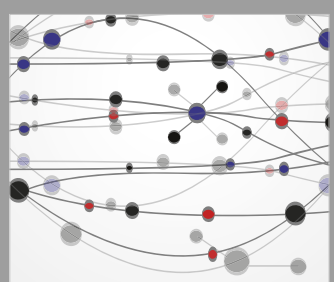

The Scientific World Journal
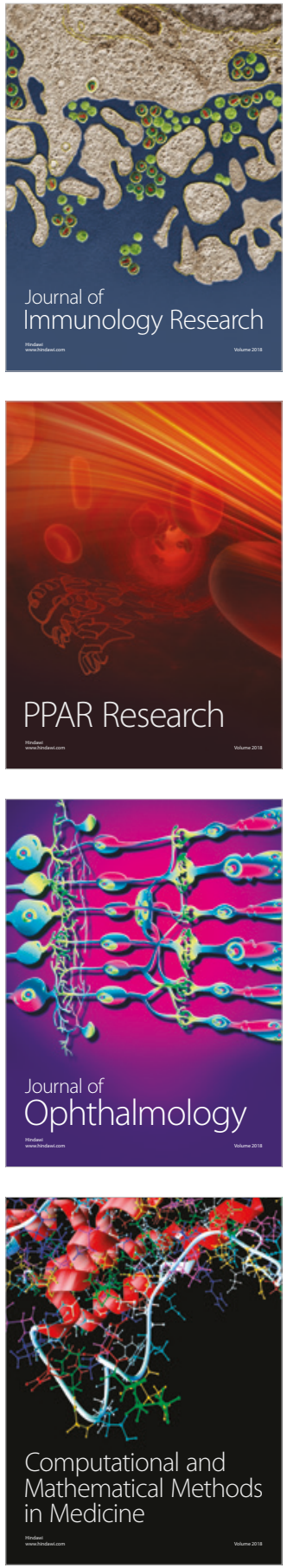

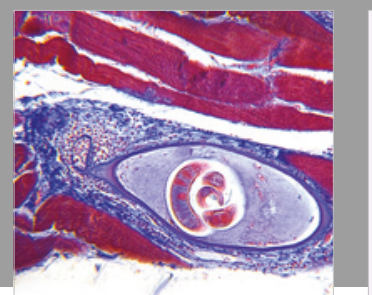

Gastroenterology Research and Practice

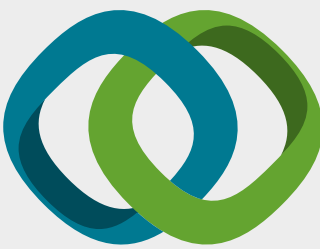

\section{Hindawi}

Submit your manuscripts at

www.hindawi.com
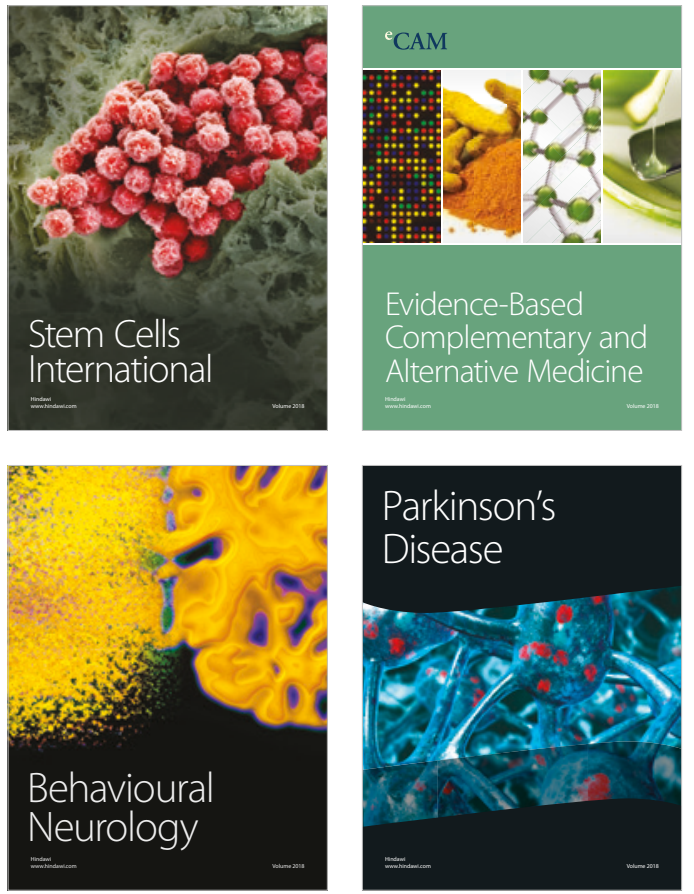

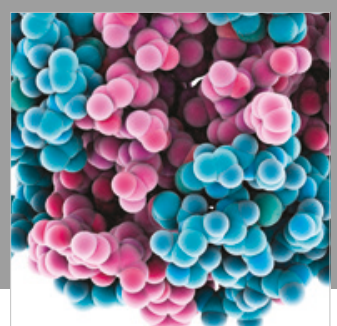

ournal of

Diabetes Research

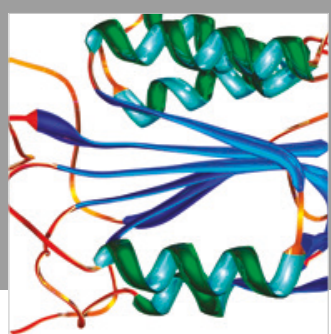

Disease Markers
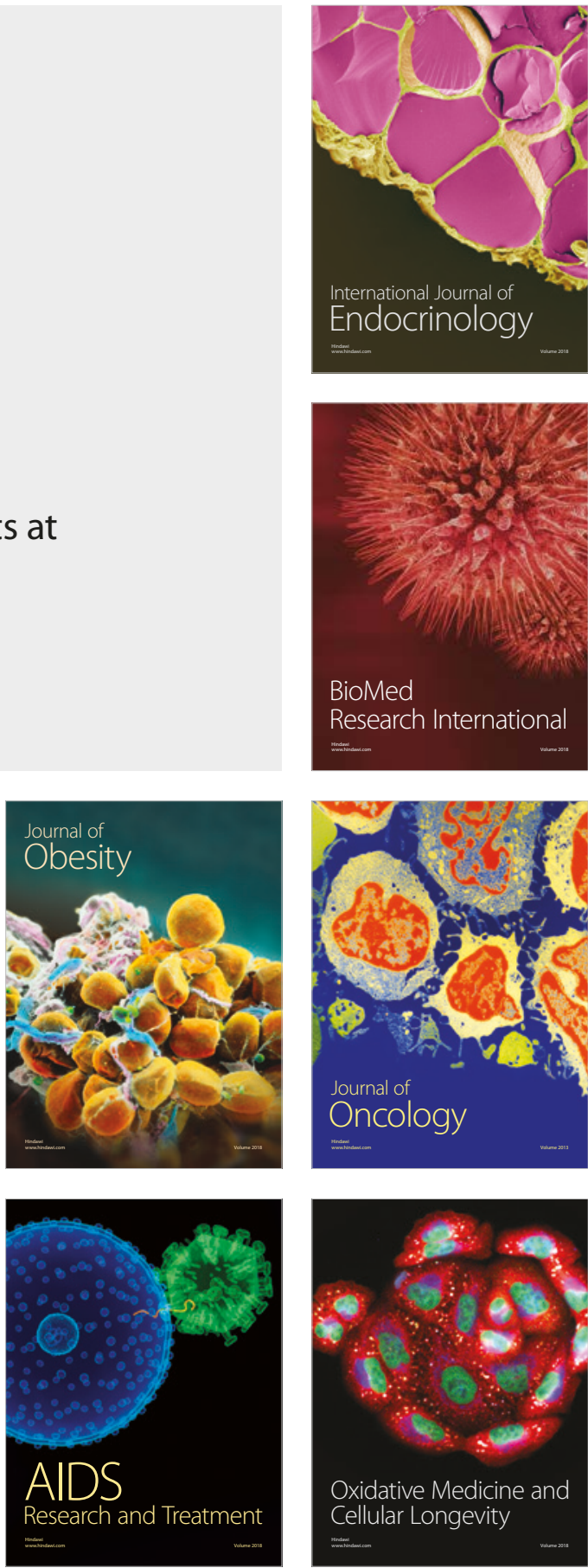